\title{
Second Harmonic Generation Using an All-Fiber Q-Switched Yb-Doped Fiber Laser and MgO:c-PPLN
}

\author{
Yi Gan, ${ }^{1}$ Xijia Gu, ${ }^{2}$ Joyce Y. C. Koo, ${ }^{2}$ Wanguo Liang, ${ }^{1}$ and Chang-qing Xu ${ }^{1}$ \\ ${ }^{1}$ Department of Engineering Physics, McMaster University, Hamilton, ON, Canada L8S 4L7 \\ ${ }^{2}$ Department of Electrical and Computer Engineering, Ryerson University, Toronto, ON, Canada M5B 2K3
}

Correspondence should be addressed to Yi Gan, gany@mcmaster.ca

Received 13 June 2008; Accepted 26 August 2008

Recommended by Hiroshi Murata

We have experimentally demonstrated an efficient all-fiber passively Q-switched Yb-doped fiber laser with Samarium doped fiber as a saturable absorber. Average output power of $3.4 \mathrm{~W}$ at a repetition rate of $250 \mathrm{kHz}$ and a pulse width of 1.1 microseconds was obtained at a pump power of $9.0 \mathrm{~W}$. By using this fiber laser system and an $\mathrm{MgO}$-doped congruent periodically poled lithium niobate (MgO:c-PPLN), second harmonic generation (SHG) output at $532 \mathrm{~nm}$ was achieved at room temperature. The conversion efficiency is around $4.2 \%$ which agrees well with the theoretical simulation.

Copyright (C) 2008 Yi Gan et al. This is an open access article distributed under the Creative Commons Attribution License, which permits unrestricted use, distribution, and reproduction in any medium, provided the original work is properly cited.

\section{Introduction}

Over the last decades, many different display technologies have been developed to replace the traditional cathode-ray tubs (CRTs), especially in large screen projection market for business and home theater. Laser-based projection display seems to be a superior alternative to existing projection systems because laser light sources can provide extremely wide color expression. Lasers have faster response time and longer life time than any lamps. Moreover, the small system size with high brightness and high power efficiency will also attract the industry $[1,2]$.

For laser-based projection systems, a high power and high efficient green laser source with compact size is now one of key components. Traditional gas- and dye-based green lasers are not suitable for this application due to their large size, inefficiency, and cumbersomeness to maintain. Although many efforts have been focused on developing semiconductor green laser diodes, the performance achieved to date is still not satisfied because of the material limitation at this wavelength region. Nonlinear frequency conversion of high-power lasers around $1060 \mathrm{~nm}$ now seems to be the most practical method for many applications requiring high quality green lasers.

Visible fiber lasers have attracted a lot of attention recently due to potential applications in the fields of indus- trial processing, bioinstrumentation, and display. Comparing with the conventional Nd:YAG solid-state lasers, the ytterbium $(\mathrm{Yb})$-doped fiber lasers have strong potential for the systems where air-cooling, long term stability and maintenance-free operation are required. Using $\mathrm{Yb}$-doped fiber lasers and nonlinear crystals to generate CW green light has already been reported [3-5]. But for laser display application, high repetition rate pulse input is more attractive for SHG process. Because the conversion efficiency is proportional to the square of input power, which means under the same average input power, the SHG conversion efficiency of a pulsed input will be much higher than that of a CW input due to the higher pulsed peak power.

In this study, we report an air-cooled simple all-fiber, passively Q-switched $\mathrm{Yb}$ doped fiber laser utilizing Smdoped fiber as an SA. Then using this fiber laser and an $\mathrm{MgO}$-doped periodically poled lithium niobate $(\mathrm{MgO}-$ PPLN) crystal, we have achieved high repetition green pulses output at room temperature. The characteristics of fiber laser output and SHG light have been investigated.

\section{All-Fiber Q-Switched Yb-Doped Fiber Laser}

Compact Yb-doped Q-switched fiber lasers have attracted significant interests as it could provide a high power, high 
efficiency and low cost source for many applications. There are different technologies to Q-switch fiber laser that includes active Q-switching using, for example, piezoelectric modulator [6], electrooptic modulator [7], and acoustic modulator [8]. The other alternative is the passive Q-switching using a saturable absorber (SA) in laser cavity that offers much simpler design as compared to the active Q-switching. Huang et al. reported recently a passively Q-switched $\mathrm{Yb}$-doped fiber laser with $\mathrm{Cr} 4+$ : YAG as an SA and achieve a high peak power of $20 \mathrm{~kW}$ [9]. However their laser consists of a Cr4+: YAG slab, three collimating and focus lenses, and a mirror that complicated substantially the laser cavity design. Dvoyrin et al. reported pulsed Yb-doped fiber lasers with Bismuthdoped fiber as an SA [10]. They succeeded in Q-switching in wide wavelength range from 1050 to $1200 \mathrm{~nm}$ with pulse width from 1 to 1.5 microseconds at a repetition rate from 10 to $100 \mathrm{kHz}$. Nevertheless, the stable pulse operation was only obtained in double-cavity design and the peak power was limited to $40 \mathrm{~W}$ at $1064 \mathrm{~nm}$. Fotiadi et al. described the dynamics of an all-fiber self-Q-switched $\mathrm{Yb}$-doped fiber laser with a samarium absorber [11]. Regular pulse operation was observed at discrete pump levels and the dynamics was attributed to polarization mode switching. It appears that though saturable absorbers have been successfully employed in Q-switching solid-state lasers, it is still an interesting research topic to achieve high repetition rate, high peak power Q-switching in Yb-doped fiber laser.

\subsection{Experiments}

The schematic of the fiber laser configuration is shown in Figure 1. It consists of two pump lasers of $975 \mathrm{~nm}$ to provide bidirectional pump, 10.5 meters of twin-core fiber, a piece of Sm-doped fiber as an absorber and one highly reflective fiber Bragg grating (FBG). The twin-core fiber has two optically contacted large cores, coated with a low refractive index polymer as shown in Figure 2. The single-mode Yb-doped active core has a diameter of $6.7 \mu \mathrm{m}$ and an NA of 0.13 . The multimode core has a diameter of $118 \mu \mathrm{m}$ with a multimode absorption coefficient of $0.77 \mathrm{~dB} / \mathrm{m}$ at the pump wavelength. The advantage of this coupling method is the ability to increase the output power from the fiber laser by coupling multiple pump sources at both ends, or by exploiting the physical separation between the pump and active paths in which the independent access to the pump path allows for power scaling by adding the pump power along the fiber length. The efficient light coupling between the pump and active cores had been described recently in [12]. The single mode Sm-doped SA was obtained from CorAcitve (Sm119) with an NA of 0.14 and a core diameter of $6.3 \mu \mathrm{m}$. Its peak absorption near $1064 \mathrm{~nm}$ was measured to be $8 \mathrm{~dB} / \mathrm{m}$.

We select $\mathrm{Sm}$-doped absorber for its fast response time [13]. In its near IR absorption band, the lifetime of upper energy level is less than 5 nanoseconds that is essential to achieve narrow pulse width and high repetition rate when used in Q-switching.

The diode pump laser has a fiber-coupled output though a multimode fiber of $105 / 125 \mu \mathrm{m}$ core/cladding diameter,

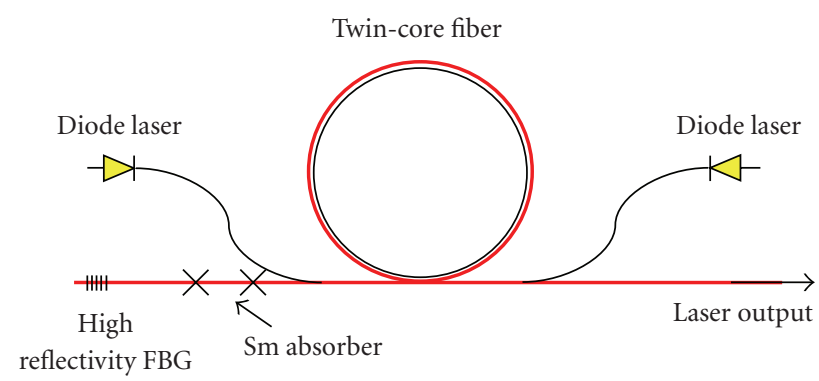

FIgURE 1: Schematic of an all-fiber Q-switched fiber laser design.

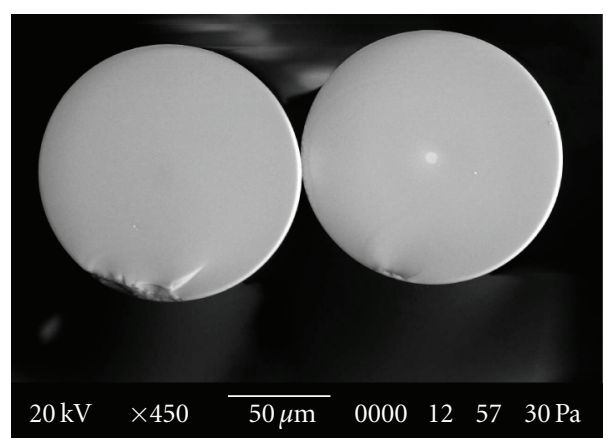

FIGURE 2: SEM image of the cross-section of the twin-core fiber coated with lower index of polymer. The left core is for pumping and the right one has a $\mathrm{Yb}$-doped core.

capable of delivering up to $6.5 \mathrm{~W}$ of optical power at $975 \mathrm{~nm}$. The high reflectivity FBG was fabricated in house with a reflectivity of $>99.9 \%$ and $\mathrm{a}-3 \mathrm{~dB}$ bandwidth of $0.6 \mathrm{~nm}$ centered at $1063.37 \mathrm{~nm}$. The cleaved end of the active fiber served as a $4 \%$ output coupler.

The temporal behavior of pulse train was recorded with an InGaAs photodiode with a bandwidth of $80 \mathrm{MHz}$ (New Focus, model: 2117) and a digital oscilloscope (Tektronix, model: TKD 3032B). The rise time of the InGaAs photodiode is about 3 nanoseconds. Optical spectra were recorded with an optical spectral analyzer (OSA) (Ando Corp. model: AQ6315E). A power meter (Coherent, model: FieldMaster-GS) was used to measure CW laser power or average power of a Q-switched pulse train.

In our laser configuration, no active cooling was required for all fiber components. Two pump diode lasers were mounted on heat dissipate plates and fiber assembly used a standard anodized aluminum fiber tray mounted on an aluminum metal plate, which separates the electrical and optical compartments in the system. The power supply, providing up to $10 \mathrm{~A}$ of current, uses a conventional fan for convection cooling.

\subsection{Results and Discussion}

Before inserting the Sm-doped SA, the CW output of the laser, assembled with same components less than that of an SA, was characterized by providing a reference for the performance of Q-switched laser output. The CW output 


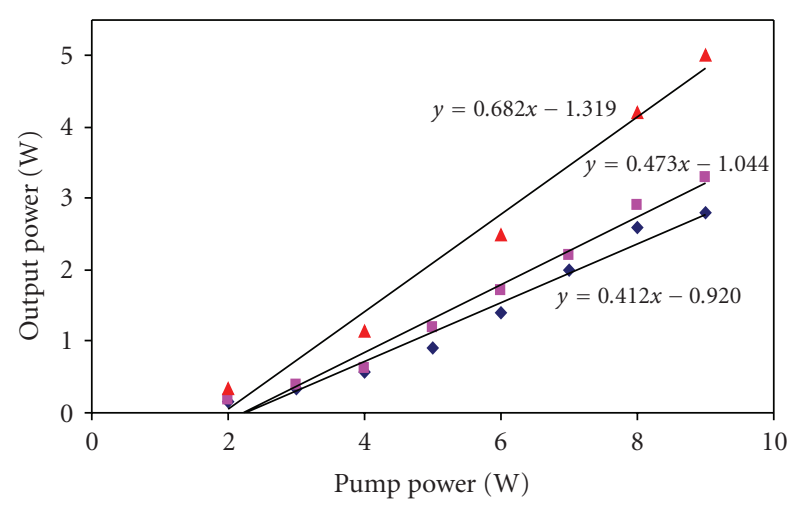

FIGURE 3: The average output power versus pump power: for CW output $(\mathbf{\Lambda})$; for Q-switched output with a $0.45 \mathrm{~m}$ of Sm-absorber $(\boldsymbol{\square})$; that with a $0.6 \mathrm{~m}$ of $\mathrm{Sm}$-absorber $(\checkmark)$.

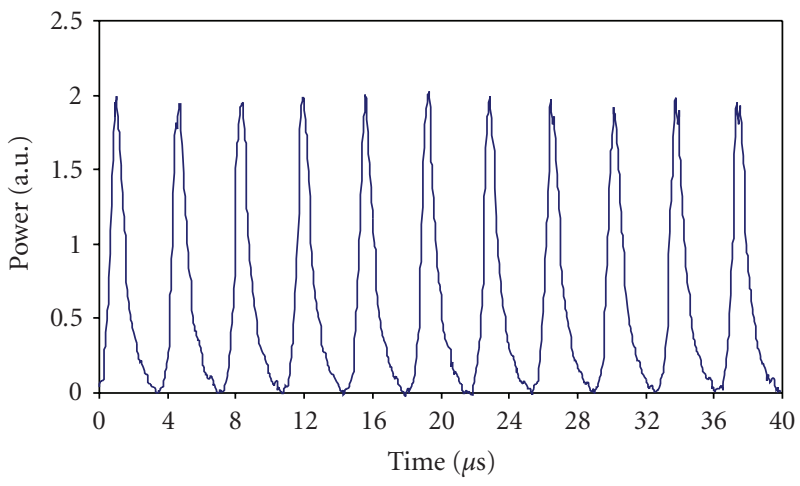

FIgURE 4: Typical Q-Switched pulse train recorded with oscilloscope at a pump power of $8.4 \mathrm{~W}$.

as a function of pump power is plotted in Figure 3. In CW operation, the laser delivered $5 \mathrm{~W}$ at a pump power of $9 \mathrm{~W}$ with a slope efficiency reached $68 \%$.

After inserting $0.6 \mathrm{~m}$ of Sm-doped SA, the Q-switched output gave an average power of $2.8 \mathrm{~W}$ at a pump power of $8.2 \mathrm{~W}$ with a power conversion efficiency of $41 \%$. The laser with a shorter $\mathrm{Sm}$-absorber fiber length of $0.45 \mathrm{~m}$ improved the slope efficiency and produced higher average power (see Table 1 for more details). The average output power of the Qswitched laser as function of the pumped power for two Smabsorber lengths is also plotted in Figure 3. The Q-switching efficiency (the ratio of the Q-switched slope efficiency to that of CW one) was $69 \%$ and $60 \%$ for $0.45 \mathrm{~m}$ and $0.6 \mathrm{~m}$ of Smabsorber, respectively, indicating that $\mathrm{Sm}$-abosrber is a good candidate for Q-switching fiber laser.

Stable Q-switched pulses were obtained when the pump power was at much higher level than the threshold value. Figure 4 displays the oscilloscope trace of the pulse train. The pulse shape exhibits a fast leading edge and a slow falling edge that is typical for the Q-switched pulse. The pulse width was measured to be 800 nanoseconds at FWHM and the pulse duration is 3.6 microseconds for a $0.6 \mathrm{~m}$ long Sm-doped fiber at $8.4 \mathrm{~W}$ pump power. The pulse-to-pulse stability was better than $\pm 5 \%$. If the pump power is increased over $9 \mathrm{~W}$,

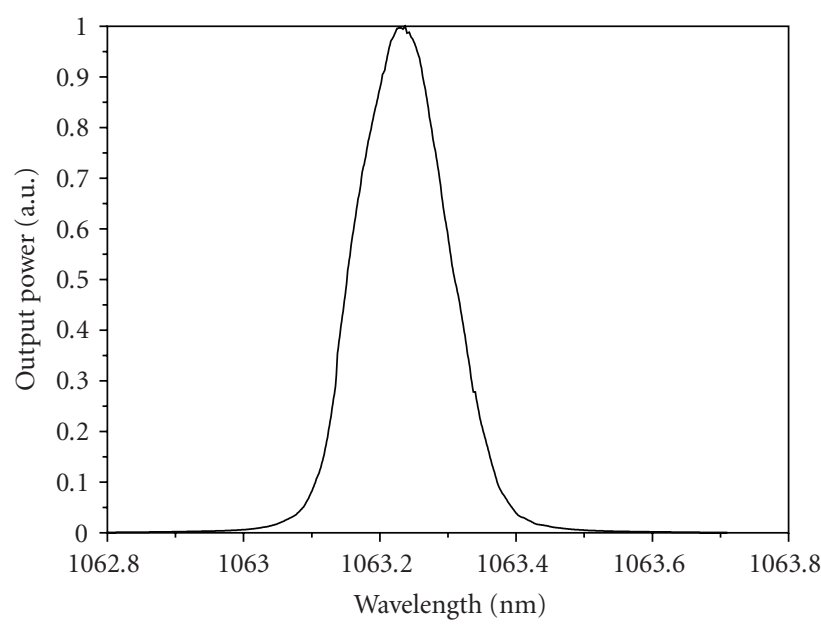

FIGURE 5: The output spectrum of the Q-switched fiber laser.

TABLe 1: Parameters of Yb-Sm Q-switched fiber laser.

\begin{tabular}{lcc}
\hline & \multicolumn{2}{c}{ Sm-absorber length } \\
& $0.6 \mathrm{~m}$ & $0.45 \mathrm{~m}$ \\
\hline Repetition rate, $\mathrm{kHz}$ & 278 & 250 \\
Pulse width, $\mu \mathrm{s}$ & 0.80 & 1.1 \\
Pulse energy, $\mu \mathrm{J}$ & 10.1 & 13.6 \\
Maximum average power, $\mathrm{W}$ & 2.8 & 3.4 \\
Slope efficiency, \% & 41 & 47 \\
\hline
\end{tabular}

the average output power that was attributed to the onset of nonlinear effects will start to decrease.

The spectrum of the Q-switched pulse output was measured by an OSA with its resolution set at $0.05 \mathrm{~nm}$. Figure 5 shows the spectrum at an average output power of $1.0 \mathrm{~W}$ for $0.45 \mathrm{~m}$ of $\mathrm{Sm}$-absorber. Its $-3 \mathrm{~dB}$ bandwidth is as broad as $0.158 \mathrm{~nm}$.

\section{Second Harmonic Generation}

Single-pass wavelength conversion by a nonlinear optical (NLO) crystal becomes one of the most attractive methods to generate coherent radiation in various spectral domains from ultraviolet to mid-infrared since QPM was first proposed in the early days of nonlinear optics [14]. Especially, as periodically poling technology became commercially available. Compared with other phase matching methods such as birefringent phase matching (BPM) technique, QPM technique allows us to use the largest nonlinear coefficient over the whole transparent spectral range of the crystal without the walk-off effect.

Among all the periodically poled crystals, periodically poled lithium niobate (PPLN) has been one of the most attractive materials due to its large second-order nonlinear coefficient, wide transparency band width covering UV to IR, mature and low cost fabrication process [15]. However, PPLN will suffer from pointing instability at medium powers and permanent photorefractive damage at high power [16]. Usually PPLN crystals have to heat to the temperature 


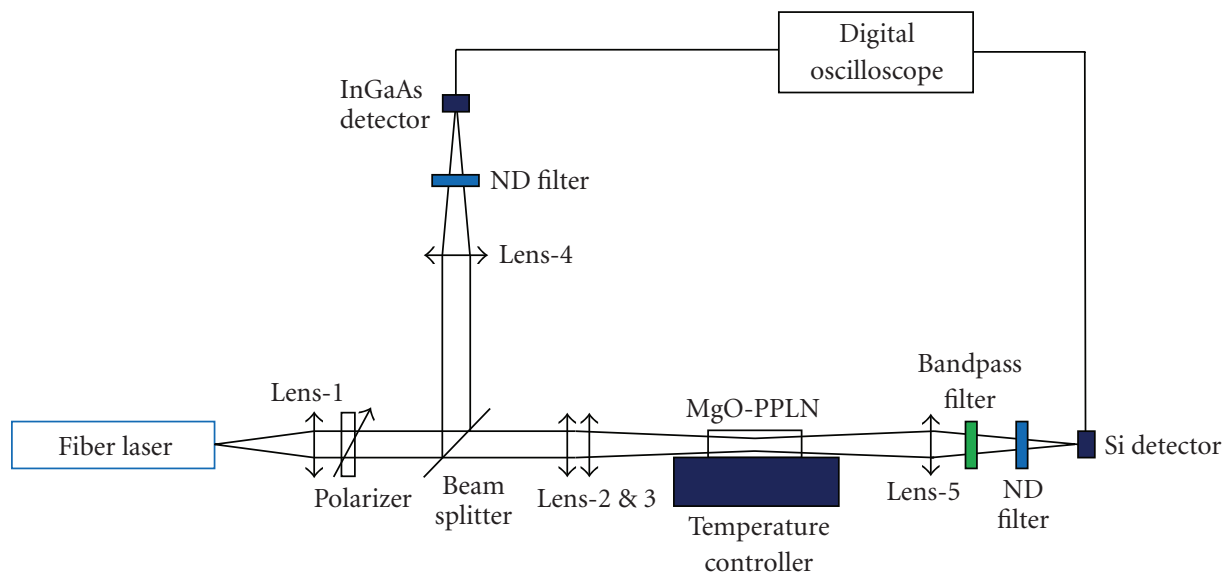

FIgURE 6: Second harmonic generation experimental setup.

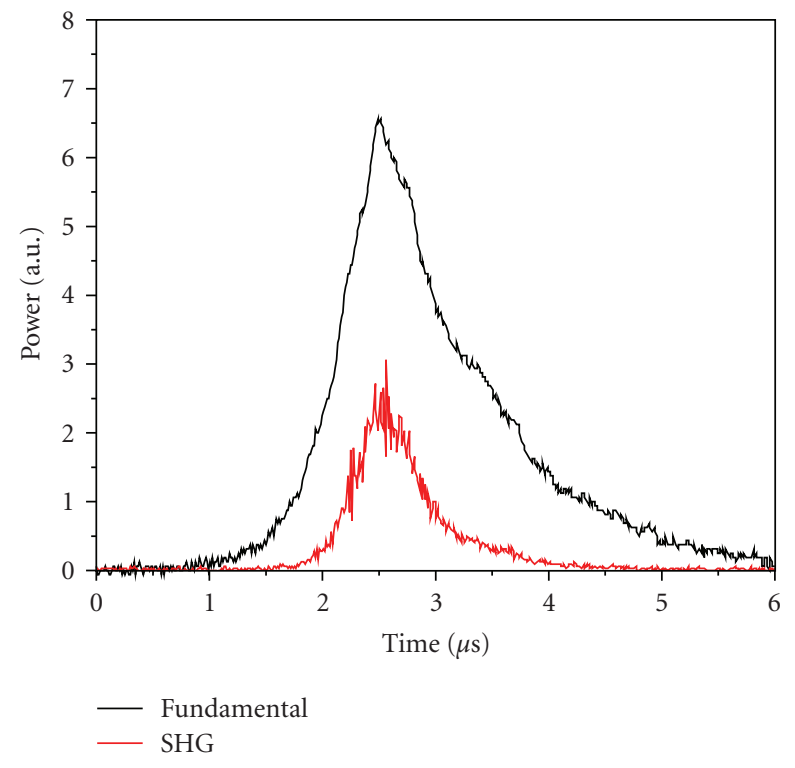

FIGURE 7: Fundamental pulse and SHG pluse.

over hundred degrees to reduce these effects. This effect also can be reduced by doping some magnesium oxide $(\mathrm{MgO})$ into the crystal during growth. Compared to pure $\mathrm{LiNbO}_{3}$ crystals, 4\%-5\% MgO:c-PPLN crystals can increase the resistance against optical damage hundred times higher, which allows devices working with high input and output power at room temperature [17].

\subsection{Experiments}

An electric-field poling technique was applied to fabricate the periodic domain structure on a $0.5 \mathrm{~mm}$ thick 3 -inch $5 \%$ $\mathrm{MgO}: \mathrm{c}-\mathrm{PPLN}$ wafer. Typically, the applied voltage is around $21 \mathrm{kV}$. A uniform periodically poled structure with periods of $6.5 \mu \mathrm{m}$ was obtained with a $50 \%$ duty ratio on the $+Z$ face.

The experimental setup is schematically shown in Figure 6. Fundamental light from Q-switched fiber laser is

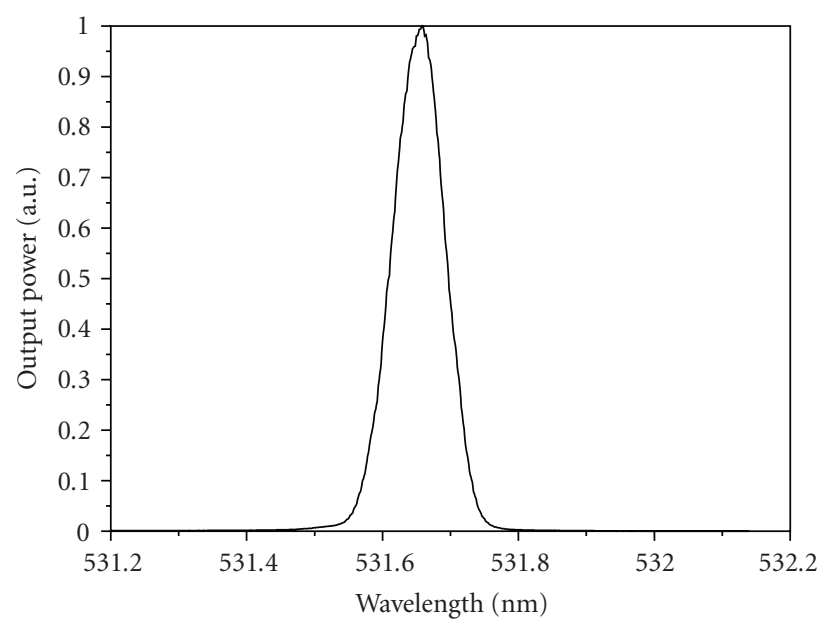

FIGURE 8: The output spectrum of SHG light.

collimated by a $10 \mathrm{x}$ objective lens (Lens-1). Since only the TM-polarization can satisfy the QPM condition, a polarizer is used to make the fundamental light's polarization parallel with the Z-direction of PPLN. A small part of fundamental light is separated by a beam splitter and focused by Lens- 4 onto an InGaAs detector (Thorlabs, model: PDA10CS). The main stream of fundamental beam is focused by two lens (Lens-2\&3). These two lenses can adjust the beam diameter to satisfy the optimum confocal condition for different sample length. In this paper, the sample length is $15 \mathrm{~mm}$ so the beam is focused to a $50 \mu \mathrm{m}$ spot radius in the center of the sample.

The sample was mounted in a temperature-controlled oven (Thorlabs, model: TC200) and the temperature was set to $34.5^{\circ} \mathrm{C}$ which optimizes the QPM condition of the sample. SHG light was focused by Lens- 5 and a bandpass filter is used to block the fundamental light. An Si detector (Thorlabs, model: PDA10A) is used for the measurement of the pulses trains. Both two detectors are connected to a digital oscilloscope (LeCroy, Model: LT224) for pulses trains recording. In this way, we can record the fundamental 


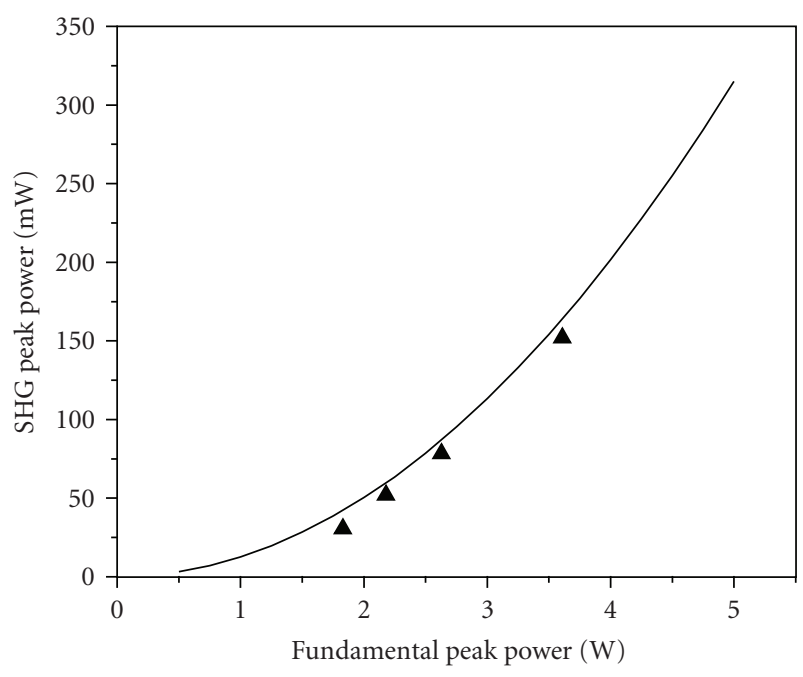

FIGURE 9: SHG peak power as a function of the fundamental peak power: the triangle dots and solid line represent experimental data and the theoretical simulation result.

pulses and SHG pulses synchronously in order to accurately compare each other. The responsivities of two detectors were calibrated by two CW DPSS lasers at $1064 \mathrm{~nm}$ and $532 \mathrm{~nm}$, respectively.

\subsection{Results and Discussion}

Figure 7 shows the comparison of a fundamental pulse and an SHG pluse. The fiber laser used in SHG experiments is connected with $0.45 \mathrm{~m} \mathrm{Sm-absorber,} \mathrm{as} \mathrm{described} \mathrm{before;}$ the fundamental pulse width is about 1.1 microseconds. Becasue the SHG power is proportional to the squre of fundamental pulse, the SHG pulse FWHM width should be 0.707 fundamental pulse. As shown in Figure 7, the SHG pulse width is about 0.75 microsecond.

Figure 8 shows the output spectrum of SHG light at $531.64 \mathrm{~nm}$. The $-3 \mathrm{~dB}$ bandwidth is about $0.09 \mathrm{~nm}$ which is slightly narrower than that of the fundamental light at $1063.25 \mathrm{~nm}$.

The SHG peak power is plotted against the incident fundamental peak power as shown in Figure 9. The SHG peak power of $152 \mathrm{~mW}$ was achieved when the fundamental peak power was $3.6 \mathrm{~W}$ (average power is $1 \mathrm{~W}$ ). The conversion efficiency (SHG pulse power/fundamental pulse power) is $4.2 \%$ which agrees with the theoretical simulation result. The conversion efficiency can be expected to be above $90 \%$ when the input fundamental peak power is in $\mathrm{kW}$ level.

\section{Conclusion}

We have demonstrated an all-fiber, high repetition rate and high peak power passively $\mathrm{Q}$-switched $\mathrm{Yb}$-doped fiber laser using Sm-doped fiber as an SA. With a pump power of $8.4 \mathrm{~W}$, the fiber laser outputs stable pulses with a pulse width of 1.1 microseconds at a repetition rate of $250 \mathrm{kHz}$, producing an average output power of $3.4 \mathrm{~W}$. All fiber connections were fusion spliced and fiber was looped in a $15 \times 12 \times 0.8 \mathrm{~cm}^{3}$ compact fiber tray. This laser design showed a significant improvement as compared with other Q-switched fiber lasers and has the following features: (a) all fiber components were splice connected, which avoided the use of any bulk optics, (b) realized bidirectional pumping using a twin-core fiber that separated pump path from the active signal path, (c) demonstrated high repetition rate in the order of $200 \mathrm{kHz}$.

Using this novel fiber laser and an MgO-PPLN wavelength conversion device, we have obtained the green laser with peak power of $150 \mathrm{~mW}$ at room temperature. The conversion efficiency is $4.2 \%$ which agrees well with the theoretical value. The MgO:c-PPLN sample is $15 \mathrm{~mm}$ long and the temperature is controlled at $34.5^{\circ} \mathrm{C}$ to satisfy QPM condition. The conversion efficiency will be higher if we can achieve high peak power for fundamental light from fiber laser. Further increasing the peak power is currently limited by the nonlinear effects in fiber laser and will be improved by the use of larger core active fiber. The allfiber configurations provide a simple, low cost and easy to manufacturing solution for high power Q-switched pulse laser that should be attractive to micromachining, medical treatment, and other applications.

\section{Acknowledgments}

This work is partially supported by Natural Sciences and Engineering Research Council of Canada (NSERC), Canadian Institute for Photonics Innovations (CIPI), Ontario Centres of Excellent (OCE), and Canadian Foundation for Innovation (CFI).

\section{References}

[1] J. Lee, Y. Mun, S. Do, et al., "Laser TV for home theater," in Projection Displays VIII, vol. 4657 of Proceedings of SPIE, pp. 138-145, San Jose, Calif, USA, January 2002.

[2] G. Niven, "Laser TV: coming to a home theatre near you," Opto \& Laser Europe, no. 142, pp. 33-34, 2006.

[3] F. J. Kontur, I. Dajani, Y. Lu, and R. J. Knize, "Frequencydoubling of a CW fiber laser using PPKTP, PPMgSLT, and PPMgLN," Optics Express, vol. 15, no. 20, pp. 12882-12889, 2007.

[4] H. Furuya, A. Morikawa, K. Mizuuchi, and K. Yamamoto, "High-beam-quality continuous wave $3 \mathrm{~W}$ green-light generation in bulk periodically poled $\mathrm{MgO}: \mathrm{LiNbO}_{3}$," Japanese Journal of Applied Physics, vol. 45, no. 8B, pp. 6704-6707, 2006.

[5] S. V. Tovstonog, S. Kurimura, and K. Kitamura, "High power continuous-wave green light generation by quasiphase matching in $\mathrm{Mg}$ stoichiometric lithium tantalate," Applied Physics Letters, vol. 90, no. 5, Article ID 051115, 3 pages, 2007.

[6] M. Leigh, W. Shi, J. Zong, J. Wang, S. Jiang, and N. Peyghambarian, "Compact, single-frequency all-fiber Q-switched laser at $1 \mu \mathrm{m}$," Optics Letters, vol. 32, no. 8, pp. 897-899, 2007.

[7] Y. Wang and C.-Q. Xu, "Modeling and optimization of Qswitched double-clad fiber lasers," Applied Optics, vol. 45, no. 9, pp. 2058-2071, 2006.

[8] Y. Kaneda, Y. Hu, C. Spiegelberg, J. Geng, and S. Jiang, "Singlefrequency, all-fiber Q-switched laser at $1550 \mathrm{~nm}$," OSA Trends in Optics and Photonics Series, vol. 94, pp. 126-130, 2004. 
[9] J. Y. Huang, H. C. Liang, K. W. Su, and Y. F. Chen, "High power passively Q-switched ytterbium fiber laser with $\mathrm{Cr}^{4+}$ : YAG as a saturable absorber," Optics Express, vol. 15, no. 2, pp. 473-479, 2007.

[10] V. V. Dvoyrin, V. M. Mashinsky, and E. M. Dianov, "Yb-Bi pulsed fiber lasers," Optics Letters, vol. 32, no. 5, pp. 451-453, 2007.

[11] A. A. Fotiadi, A. S. Kurkov, and I. M. Razdobreev, "Dynamics of all fiber self-Q-switched Ytterbium/Samarium laser," in Proceedings of the Laser and Electro-Optics/Quantum Electronics and Laser Science Conference (CLEO/QEC '07), pp. 1-2, Baltimore, Md, USA, May 2007, paper CMC4.

[12] X. J. Gu and Y. Liu, "The efficient light coupling in a twincore fiber waveguide," IEEE Photonics Technology Letters, vol. 17, no. 10, pp. 2125-2127, 2005.

[13] B. Wu and P. L. Chu, "Fast optical switching in $\mathrm{Sm}^{3+}$-doped fiber," IEEE Photonics Technology Letters, vol. 8, no. 2, pp. 230232, 1996.

[14] J. A. Armstron, N. Bloembergen, J. Ducuing, and P. S. Pershan, "Introductions between light waves in a nonlinear dielectric," Physical Review, vol. 127, no. 6, pp. 1918-1939, 1962.

[15] K. K. Wong, Properties of Lithium Niobate, INSPEC, Edison, NJ, USA, 2002.

[16] G. D. Miller, R. G. Batchko, W. M. Tulloch, D. R. Weise, M. M. Fejer, and R. L. Byer, " $42 \%$-efficient single-pass cw secondharmonic generation in periodically poled lithium niobate," Optics Letters, vol. 22, no. 24, pp. 1834-1836, 1997.

[17] D. A. Bryan, R. Gerson, and H. E. Tomaschke, "Increased optical damage resistance in lithium niobate," Applied Physics Letters, vol. 44, no. 9, pp. 847-849, 1984. 

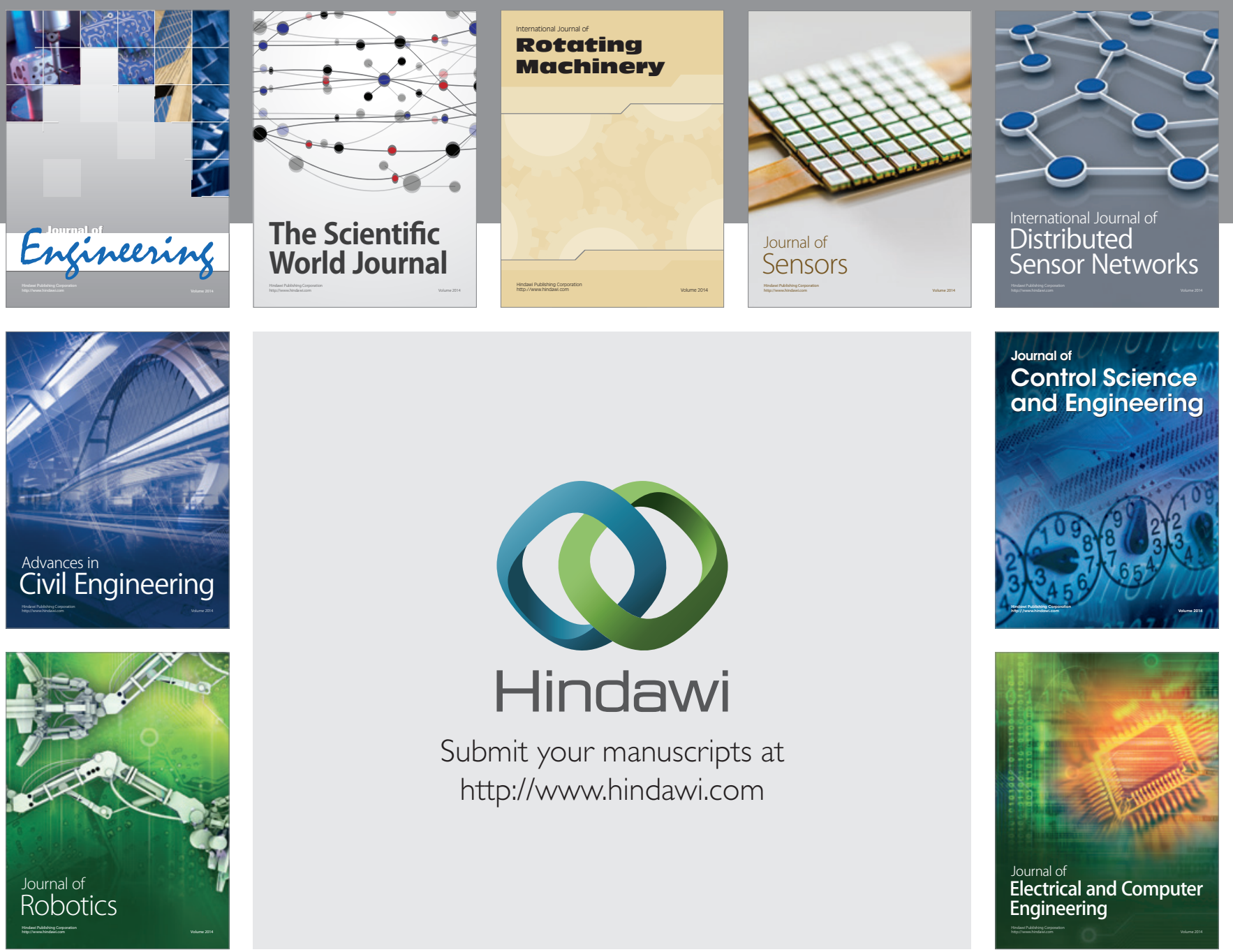

Submit your manuscripts at

http://www.hindawi.com
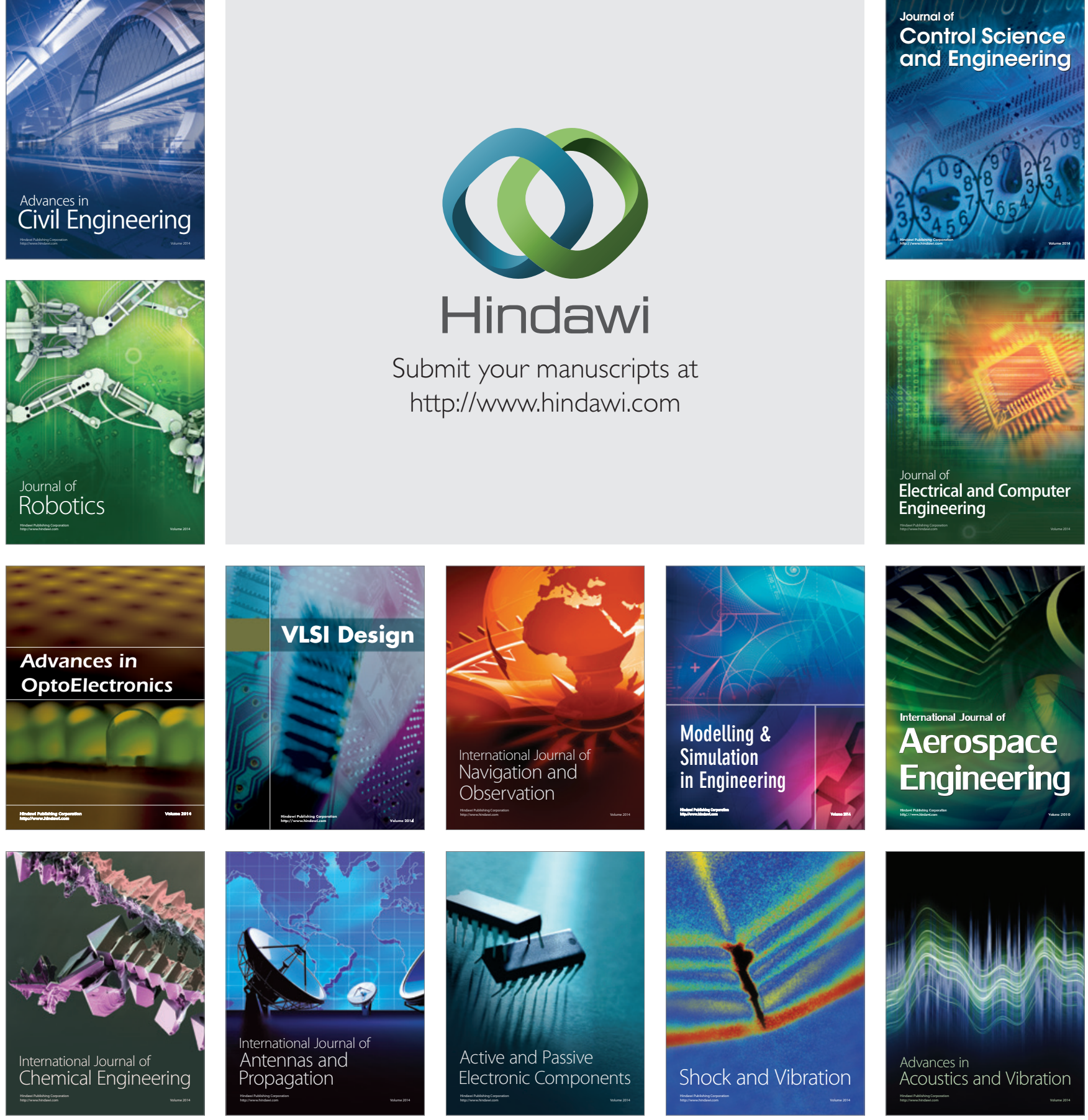